\title{
Corinne Duboin, Claudine Raynaud, eds., Diaspora, Cultures of Mobilities, "Race": African Americans and the Black Diaspora
}

\section{Sarah Fila-Bakabadio}

\section{(2) OpenEdition}

\section{Journals}

Édition électronique

URL : https://journals.openedition.org/transatlantica/9182

DOI : 10.4000/transatlantica.9182

ISSN : $1765-2766$

Éditeur

Association française d'Etudes Américaines (AFEA)

Référence électronique

Sarah Fila-Bakabadio, "Corinne Duboin, Claudine Raynaud, eds., Diaspora, Cultures of Mobilities, "Race": African Americans and the Black Diaspora », Transatlantica [En ligne], 1 | 2017, mis en ligne le 16 octobre 2018, consulté le 23 mai 2021. URL : http://journals.openedition.org/transatlantica/9182 ; DOI https://doi.org/10.4000/transatlantica.9182

Ce document a été généré automatiquement le 23 mai 2021

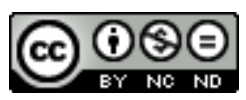

Transatlantica - Revue d'études américaines est mise à disposition selon les termes de la licence Creative Commons Attribution - Pas d'Utilisation Commerciale - Pas de Modification 4.0 International 


\title{
Corinne Duboin, Claudine Raynaud, eds., Diaspora, Cultures of Mobilities, "Race": African Americans and the Black Diaspora
}

\author{
Sarah Fila-Bakabadio
}

\section{RÉFÉRENCE}

Corinne Duboin, Claudine Raynaud, eds., Diaspora, Cultures of Mobilities, "Race": African Americans and the Black Diaspora, Montpellier, Presses universitaires de la Méditerranée, Collection « Horizons anglophones », 2016, 346 pages, ISBN : 9782367812205, $34 €$

1 L'ouvrage Diaspora, Cultures of Mobilities, "Race", publié en 2016 clôt un triptyque du même nom et fait suite à une série de trois conférences organisées aux États-Unis puis en France entre 2011 et 2013. Le premier volume (Diasporas and Cultures of Migrations) était dédié à la conceptualisation de la notion de diaspora et son insertion dans le champ universitaire. Le second, publié en 2015, (Diasporas, Memory and Intimacy) abordait la migration du concept de diaspora.

2 Dans ce dernier ouvrage pré-édité en 2015 sur le site PoCOPages, les éditrices et les auteurs reviennent sur la diaspora africaine vue depuis l'espace restreint de l'identité afro-américaine. L'objet est d'analyser l'articulation entre identité, race et diaspora africaine. Cette dernière est à la fois prise comme un champ d'études (les African Diaspora Studies) et comme un espace historique et épistémique où se jouent des dynamiques identitaires réelles ou imaginaires. 


\section{Résumé et propos de l'ouvrage}

3 La démarche de l'ouvrage est ambitieuse: discuter, en quatre parties et quatorze contributions, du champ des African Diaspora Studies à travers la reconceptualisation de l'identité afro-américaine en termes de "fluidité, de multiplicité, et de contingence » (13). Les éditrices proposent de penser un local africain-américain à travers un global afro-descendant et inversement. La difficulté est ici de penser ces circulations (ici nommées « mobilités ») tout en travaillant un objet qui est, lui, ancré dans une histoire, un territoire (les États-Unis) et, originellement, dans un champ (les Black Studies).

4 La seconde difficulté relevée par les auteurs et les éditrices est méthodologique. Comment développer une pensée critique et théorique issue de la diaspora africaine tout en la prenant comme objet d'étude ? Comment le faire sans nier la pertinence d'une histoire proprement africaine-américaine? Pour répondre à ces questions, Corinne Duboin et Claudine Raynaud reprennent la perspective de la sociologue Avtar Brah qui envisage la diaspora comme un espace construit par l'intersection (voire par l'intersectionnalité) des cultures, des identités et des trajectoires. Comme Brah, les éditrices postulent que les phénomènes de "dis/location" (c'est-à-dire de rapprochements, d'éloignement, de distanciation et de croisement) ont généré la diaspora autant que l'identité africaine-américaine. Dès lors, cette identité n'est plus la résultante de la seule présence noire aux États-Unis mais elle est le "processus d'un mouvement et d'une médiation » (Gilroy $1993: 19$, cité 13) entre populations noires. Les éditrices réactivent un courant visible depuis la fin des années 1990, notamment illustré par l'expansion des départements d'études diasporiques et des Africana Studies : l'histoire africaine-américaine est moins celle d'une minorité américaine que le rhizome nord-américain d'un vécu global noir.

Dans l'ouvrage, ces thèmes sont abordés à travers des parcours de vie comme ceux de l'écrivain James Baldwin ou des abolitionnistes Jeffrey Brace et Charles Bowles, ou par des études de cas comme le chapitre de Michael Gomez sur le lien entre blackness et diaspora vu depuis le Moyen-Orient.

On sent au fil des pages de l'introduction une compréhension fine de l'abondante littérature sur le champ comme une grande maîtrise des concepts qui le structurent. De ce fait, on aurait aimé voir un positionnement plus ferme des éditrices allant au-delà de l'affirmation de la multiplicité de l'identité afro-américaine (17) résolument ancrée dans la diaspora africaine et faisant le lien avec les deux publications précédentes. En effet, apparait dans cet ouvrage la tension continue entre, d'un côté, la construction d'un «en-commun » noir, pensé à travers la racialisation et le racisme subis par les populations d'ascendance africaine et de l'autre, la défense d'une subjectivité africaineaméricaine. Comme le notent ici Mark Reid et Christine Vogt-William, en participant à la construction d'une vision diasporique africaine du monde, les Africains-Américains ont dû réévaluer leur identité face à ces " autres autruis » que sont les Africains et les Afro-descendants du monde. Ce repositionnement a lui-même fécondé des œuvres plastiques et littéraires et a initié des mouvements politiques et intellectuels comme le panafricanisme (voir Mangeon 2010). 


\section{Structure du livre}

7 Les quatre parties du livre offrent leur circulation propre: de la théorisation de la diaspora à l'engagement militaire des Africains-Américains en passant par l'expatriation puis la représentation du sujet noir. Les auteurs y abordent des questions récurrentes qui traversent les African Diaspora Studies comme d'autres area studies : quel parallèle existe-t-il entre la diaspora africaine et les diasporas historiques? Quelle différence entre les African Diaspora studies et le panafricanisme? Y a-t-il un écart sémantique entre " Afro-descendants » » et " Africains de la diaspora », etc.

8 La première partie, intitulée "Theorizing Diaspora, Blackness \& Post-Raciality" aborde la conceptualisation de la diaspora africaine en tant que modèle. Les auteurs dont certains, comme Michael Gomez, y ont déjà longuement réfléchi, discutent de l'historicité et de la plasticité du concept. Ashraf Rushdy propose par exemple une généalogie des African Diaspora Studies qui témoigne des difficultés heuristiques et pratiques des précurseurs de ce champ. Dans ce très bon chapitre, il montre qu'entre les années 1960 et 1970, les African Diaspora Studies peinent à se constituer. Certains, comme l'historien de l'université de Howard, Joseph E. Harris, mettent l'accent sur l'origine africaine («African base», 37) des peuples noirs dispersés. Pour Harris, la diaspora africaine s'appuie sur l'idée d'un retour physique, spirituel et/ou psychologique des Afro-descendants vers la "terre mère " Afrique. Pour d'autres, comme Walter Rodney et St Clair Drake, si l'Afrique est un commun minimal entre Africains et Afro-descendants, ils insèrent leurs réflexions dans une lutte postcoloniale des "peuples de couleur» (Walter Rodney cité par Rushdy 39-40). Tous s'accordent pour refuser le «panafricanisme racial» (St Clair Drake cité 39) de Maulana Karenga qui met l'accent sur une authenticité imaginée et donc biaisée. Par ce travail, Rushdy montre que les questions soulevées lors des conférences fondatrices d'Addis-Abeba (1965) et de Washington (1979) restent d'actualité. Penser les diasporas africaines (autant que la diaspora africaine) signifie interroger le lien avec un territoire des origines autant que la centralité de la Traite transatlantique comme événement fondateur de cet espace.

9 Michael Gomez teste lui la notion de diaspora africaine. À la suite de Paul Tiyamba Zeleza (2005), il propose de décentrer le regard de l'Atlantique noir. Il choisit de l'observer depuis les sociétés esclavagistes et post-esclavagistes du Golfe Persique, du Maghreb (en l'espèce les Émirats et le Maroc) et de la République dominicaine. Malgré des contextes très différents, il argue que le rejet du «noir » dans ces sociétés constitue en fait des stratégies de «neutralisation » (54) de toute référence à l'Afrique ou à une africanité qui imprégnerait ces sociétés. Si la comparaison entre le Moyen-Orient et la République dominicaine semble quelque peu difficile à tenir, il reste que la notion d'africanité, recherchée ou rejetée, pose des questions récurrentes aux African Diaspora Studies: doit-on parler de diaspora noire (pensée à travers la catégorie de race) ou africaine (pensée à travers la valorisation d'une trace africaine)? Que signifie l'« africanité »?

10 Les deux articles de Lisa Veroni-Paccher et Mark Reid réorientent le débat sur la race et le racisme. Ils évoquent l'impact de la catégorie de race dans la construction de l'espace social et politique aux États-Unis, en France, en Italie et en Angleterre. Tous deux prennent comme point de départ l'Amérique d'Obama, celle qui, dans le sillage de sa première élection présidentielle, a fait émerger le concept de postracialité. Ces 
auteurs proposent de comparer des trajectoires (celles de femmes et d'hommes politiques noirs comme l'évolution des représentations médiatiques des noirs) qui témoignent d'une visibilité inédite des populations noires. Ils parlent brièvement du "post-black », autre concept du dépassement de la race qui ne semble toujours pas faire recette. Ils arguent que, d'un côté, l'arrivée de migrants d'Afrique subsaharienne et, de l'autre, l'émergence d'identités genrées et LGBTQ ont obligé les sociétés occidentales, (notamment les anciennes puissances coloniales et esclavagistes) à repenser leur ordre hiérarchique racial fondé sur la dichotomie noir/blanc. À mon sens, la question de la négociation noir/blanc sous l'influence d'autres identités n'a que peu d'influence sur le schéma racial américain, britannique, voire français. La plasticité de la catégorie « noir» se joue réellement dans les relations entre populations noires. Les AfricainsAméricains, descendants de personnes mises en esclavage, doivent désormais composer avec d'autres Africains-Américains issus de migrations récentes dont Obama est une incarnation. Mais ces adaptations n'ont pas d'effets sur les représentations sociétales de ces individus. Ils sont avant tout perçus comme des Noirs. La question est plutôt de savoir comment ces derniers cherchent, notamment grâce à la diaspora africaine, à se représenter comme un centre dont l'existence n'est pas uniquement liée à la place que la majorité lui attribue dans la société américaine.

11 La seconde partie ("African Americans 'Race' and/in War ») aborde la présence noire dans l'armée. Cette partie est la seule dont le lien avec le thème du livre n'apparaît pas de prime abord. Malgré la qualité des contributions qui éclairent plusieurs aspects de l'engagement militaire afro-américain, ces dernières relèvent à mon sens plus de l'histoire africaine-américaine classique que de Diaspora Studies. Le combat des abolitionnistes Jeffrey Brace et Charles Bowles ou le développement d'une conscience racisée chez les vétérans précurseurs du Mouvement pour les Droits Civiques ne s'inscrivent pas dans la diaspora mais dans un combat politique national. Seule exception, le chapitre de Christine Vogt-William sur l'expérience des GIs africainsaméricains en Allemagne telle que représentée dans deux ouvrages de fiction. VogtWilliam montre la confrontation des soldats africains-américains avec une autre réalité de populations racisées. En ce sens, son travail poursuit celui de Tina Campt (2012) tant sur les représentations croisées des populations noires en Allemagne (AfricainsAméricains et Afro-Allemands) que sur la circulation atlantique des objets symboles des migrations des hommes (images, livres, etc.).

12 La troisième partie s'intéresse aux « expatriations » et aux mouvements diasporiques («Diasporic Motions»). Les auteurs évoquent les trajectoires d'artistes africainsaméricains qui, comme James Baldwin, se sont installés en Europe pour parler « de loin " de l'expérience africaine-américaine. Cette partie, plus littéraire, aborde la construction d'un "regard éloigné » (Levi-Strauss) qui les autorise à " désincarcérer » l'histoire afro-américaine du récit étatsunien et à en révéler les connexions avec l'Afrique et l'Europe. La forme artistique (livre, peinture) devient alors une médiation entre une histoire afro-américaine et une histoire noire ; entre la traite transatlantique, les années 1960 de la ségrégation et le contemporain d'une société supposément postraciale. La distance leur permet de créer de nouvelles solidarités (Africains-Américains, Africains colonisés, Noirs en France) et d'interroger les notions de race des deux côtés de l'Océan atlantique. Cet éloignement aide aussi à déconstruire tout fantasme récurrent d'une relation exclusive entre une Afrique-racine et un espace flou que serait la diaspora africaine. Dans cette partie de l'ouvrage, on voit comment plusieurs artistes dont Langston Hughes et Richard Wright utilisent leurs séjours en France pour étendre 
l'expérience afro-américaine de la diaspora africaine à une autre société et tenter de la croiser avec des celles des colonialisés. On saisit comment leurs « détours » en Europe ne sont en fait pas des digressions par rapport à l'histoire afro-américaine, mais une manière de la construire autant que le Mouvement pour les Droits Civiques ou, aujourd'hui, Black Lives Matter.

13 La quatrième et dernière partie ("Re-presenting the Black Subject »), explore ce que Alan Rice décrit comme la « praxis culturelle de l'Atlantique noir » (232) soit les usages artistiques contemporains noirs (américains, britanniques, cubains) qui travaillent la mémoire de l'esclavage. À l'instar de l'exposition Mining the Museum de Fred Wilson, l'art contemporain africain-américain évoque l'absence des histoires noires dans les musées occidentaux. Entre un travail sur la résurgence des traces d'Afrique dans les œuvres de Lubaina Himid ou Marie Magdalena Campos-Pons qui utilisent l'archive pour recomposer une narration africaine-américaine où les Africains sont humanisés et la réinterprétation américaine de la figure de la femme griot dans l'œuvre de Renée Stout, les appropriations culturelles deviennent des instruments de mise en valeur d'une histoire morcelée. Si les artistes mentionnés sont moins connus que Kara Walker ou Carrie Mae Weems, ils parlent eux aussi de la matérialité de la mémoire vue à travers sa confrontation à un récit national américain. C'est ce que Alan Rice décrit comme la "guerilla memorialisation » ou comment l'art révèle à la fois l'invisibilité plastique de la mémoire africaine-américaine (l'absence d'objets dans les expositions) et oblige ces artistes à en défendre la moindre trace. Dans une perspective quelque peu herskovienne, la mémorialisation passe par la quête de survivances qui ouvrent vers des relations diasporiques à l'Afrique. C'est une posture un peu ancienne même si on peut reconnaître qu'elle reste un moyen pour ces artistes de « négocier » leurs relations à la diaspora.

14 L'intérêt de cette dernière partie est de discuter de la re-narration par l'image. Les artistes plasticiens y utilisent le visuel pour renverser le regard et jouer sur le basculement de l'histoire: on change de point de vue, de celui du maître à celui de l'esclave, on passe alors d'un récit où les esclaves sont invisibles à celui, plus complexe, de leur hybridation lors du voyage dans le passage du milieu. Le très bon article de Heather Smyth ouvre des pistes intéressantes et fait écho à celui de Michael Gomez comme une sorte de conclusion aux débats portés par ce livre. Il invite lui aussi à un décentrement. Smyth aborde les coalitions entre Afro-Canadiens et Afro-Américains au Canada. Elle montre comment des solidarités se forment à partir d'une même expérience de racialisation dans un espace qu'elle nomme le «Black Pacific». Si le décentrement est un thème à la mode, il ouvre peut-être ici vers une distanciation visà-vis de la traite transatlantique comme événement structurant, si ce n'est fondateur, de la diaspora. Car comme le montrent ici plusieurs auteurs, les acteurs ont eux-mêmes inventé/pratiqué d'autres circulations en Asie, en Amérique Latine ou dans l'Océan pacifique ${ }^{1}$. Ces mobilités alternatives participent également de la construction des African Diaspora Studies, et ce, y compris dans leur relation à l'histoire afro-américaine. Dès lors, on peut étendre la réflexion de Smyth sur des diasporas africaines « issues de » mais « distinctes de » l'Atlantique noir. 


\section{Critiques}

15 Deux points annoncés dans l'introduction et présents en filigrane de la plupart des contributions auraient pu être débattus plus avant.

16 Le premier est un postulat: après la Seconde Guerre mondiale, la race s'efface pour laisser place aux études africaines puis, dans les années 1960, pour renouer historiquement et culturellement avec l'Afrique (13). À mon sens, et comme le montrent les contributions de ce livre, la catégorie de race n'a jamais cessé d'organiser le champ social comme le champ académique aux États-Unis. Si l'introduction des African Diaspora Studies a placé le lien culturel avec l'Afrique au centre de l'analyse, il reste que la race est une composante essentielle de l'idée d'« africanité ». Les African Diaspora Studies n'échappent pas à la quête d'une visibilité physique des racines qui veut que la couleur noire signale une ascendance africaine. Cet imaginaire participe de la construction du champ autant que la reconnaissance de l'hybridité intrinsèque de l'identité africaine-américaine. Les « oscillations » (13) entre «black» et «African » ne concernent finalement que les mouvements nationalistes pris dans les débats entre une stratégie révolutionnaire et culturaliste.

17 Par ailleurs, la race n'a pas «laissé place aux études africaines» (13). Les études africaines sont apparues au début du $20^{\mathrm{e}}$ siècle et se sont institutionnalisées entre les années 1950 et 1960. Leur développement n'est pas lié aux études afro-américaines telles que portées par W.E.B. Du Bois ou Carter G. Woodson ni, plus tard, aux Black Studies. Elles ont suivi leur propre voie avant de rencontrer celle des African American Studies dans les années 1990. En effet, lorsqu'en 1922, William Leo Hansberry crée le premier cours en histoire africaine à l'université Howard, ce cours n'est pas intégré au département d'études afro-américaines mais au département d'histoire. Dans les années 1960, les Black Studies ont, elles, créé un espace pour l'histoire africaineaméricaine dans un cadre temporel et géographique unique : les États-Unis. Ce n'est que dans les années 1990, qu'une convergence apparaît, notamment sous l'influence des African Diaspora Studies qui transforment les départements d'études africainesaméricaines en Africana Studies. L'insertion des Black Studies dans les Africana Studies continue de générer des débats parfois vifs sur la perte d'une identité académique acquise par la lutte contre la ségrégation et la scission de facto avec les African Studies. Effectivement, les études diasporiques ont créé un lien entre ces espaces apparemment étanches mais pas au point d'en initier la fusion ou le remplacement de l'une par l'autre.

18 Le second point relève d'une attente suscitée par plusieurs contributions où les notions de post-racialité et même de post-blackness sont évoquées. La post-racialité a connu un certain succès après l'élection de Barack Obama en 2008 mais a rapidement été critiquée (voir pour exemple David Golberg, 2016). D'aucuns l'ont, à raison, jugée inopérante dans une société américaine où les populations noires restent la cible d'un racisme structurel. D'un point de vue théorique, je partage l'avis de Robert GoodingWilliams et Charles W. Mills (2014), le postracial est une hyperbole des premières années de l'ère Obama qui s'articule sur une vision idéalisée de la société américaine. Les éditrices rappellent d'ailleurs qu'il est un "geste utopique » (15), une temporalité sans effet réel sur les hiérarchies raciales aux États-Unis. Dès lors, on s'interroge sur son insertion dans un argumentaire qui fonctionne très bien sans cette référence. Si les 
auteurs et éditrices souhaitaient discuter du moment post-racial comme d'un tournant pour les African Diaspora Studies, il aurait été souhaitable d'en parler plus longuement.

Malgré ces quelques critiques, cet ouvrage, comme d'ailleurs les précédents, est un bon état des lieux et une bonne synthèse des African Diaspora Studies au début du $21^{\mathrm{e}}$ siècle. Il pose les questions récurrentes au champ, en montre les tensions et les continuités et ouvre plusieurs pistes intéressantes tant pour le lecteur averti que néophyte. Dans le contexte contemporain d'émergence de nouvelles Area studies comme les études décoloniales et les Black Studies européennes, il semble intéressant de saisir comment les African Diaspora Studies parviennent à perdurer.

\section{BIBLIOGRAPHIE}

CAMPT Tina, Image Matters: Archive, Photography, and the African Diaspora in Europe, Durham, Duke University Press, 2012.

GOLDBERG David, Are We All Postracial Yet?, Malden, Polity Press, 2016.

GOODING-WILLIAMS Robert et Charles W. Mills, « Race In A “Postracial” Epoch », Du Bois Review, $11: 1,2014,1-8$.

LÉVI-STRAUSS Claude, Le Regard éloigné, Paris, Plon, 1983.

MANGEON Anthony, La Pensée noire et l'Occident : De la bibliothèque coloniale à Barack Obama, Paris, Sulliver, 2010.

ZELEZA Paul Tiyamba, « Rewriting the African Diaspora: Beyond the Black Atlantic », African Affairs. 104 (414) : 35-68, 2005.

\section{NOTES}

1. Voir à ce sujet les travaux de Roberto Castillo sur les Africains dans la Chine contemporaine.

\section{INDEX}

Thèmes : Recensions 
AUTEUR

SARAH FILA-BAKABADIO

Université de Cergy-Pontoise 